论 文

\title{
EB 病毒感染对肝脏移植术后并发多发性骨髓瘤的 影响
}

张业伟 ${ }^{(1)}{ }^{*}$ ，赵何伟 ${ }^{(1 \dagger}$ ，何侠 ${ }^{(2)}$ ，郑苏文 ${ }^{(1)}$ ，王太洪 ${ }^{(1)}$, 严栋梁 ${ }^{(3)}$, 孙静锋 ${ }^{(1)}$, 鲁翔 ${ }^{(4)}$, 闻鉴非 ${ }^{(1)}$, 刘允怡 $^{(3)}$

(1) 南京医科大学附属江苏省肿瘤医院肝胆胰外科, 南京 210009;

(2) 南京医科大学附属江苏省肿瘤医院放疗科, 南京 210009;

(3) 南通大学第二附属医院普外科, 南通 226001;

(4) 南京医科大学附属第二医院老年科, 南京 210011;

(5) 香港中文大学威尔斯亲王医院, 香港

$\dagger$ 同等贡献

* 联系人, E-mail: zhangyewei@njmu.edu.cn

收稿日期: 2011-12-20; 接受日期: 2012-06-27

国家自然科学基金(批准号: 61141013)、江苏省自然科学基金(批准号: SBK201120268)、江苏省医学重点人才计划(批准号: RC2011090)、 江苏省“333”工程(批准号: 2011III-2640)资助项目

摘要 肝移植术后患者细胞免疫功能低下, 容易引起 $\mathrm{EB}$ 病毒等多种病毒感染. $\mathrm{EB}$ 病毒是 一种 $\gamma$ 亚科疮疹病毒, 它和人类很多恶性疾病有关, 特别是上皮和淋巴起源的肿瘤. 异常的 CD40-CD40L 相互作用或异位的 CD40L 表达被认为与骨髓瘤的发展密切相关. 本研究探讨 EBV 感染对肝移植术后并发多发性骨髓瘤(MM)细胞表型和生物学的影响及机制。通过流式 细胞术检测 10 例肝移植术后有 EBV 感染病史并发多发性骨髓瘤患者的 MM 新鲜标本, 结果 显示 5, 8, 9 和 10 号标本有 CD40 和 CD40L 共表达现象, 其中 5 号标本呈 CD40 和 CD40L 高 度共表达. 用 EB 病毒体外感染 MM 细胞株 RPMI8826, 通过检测 EB 病毒的病毒抗原 LMP1 和 EBNA-2, 发现 EB 病毒能体外感染 MM 细胞. 感染后细胞在 mRNA 和蛋白水平上调 $\mathrm{CD} 40 \mathrm{~L}$ 表达, 并且这种上调可能参与感染后细胞抗调亡效应。而且, 共聚焦显微镜定位检测 表明, CD40, CD40L 和 LMP1 分子能在感染后细胞表面两两共聚集. 此外, EB 病毒感染能上 调 RPMI8226 细胞趋化因子受体 CXCR4 表达, 并且在趋化因子 SDF-1a 存在的情况下, 感染 后细胞迁移率提高. 细胞的抗调亡能力和迁移扩散能力是肿瘤恶性特征的重要表现, EB 病毒 作为 MM 起源的一种高危因素, 可能通过体内感染生发中心 B 细胞使其转化产生抗调亡的效 应和提高细胞向外周血的迁移能力, 从而参与 $\mathrm{MM}$ 的发生和发展.

肝移植术后患者细胞免疫功能低下 ${ }^{[1]}$, 特别是应 用大剂量的激素冲击治疗后, 易引起多种病毒感染,
如 EB 病毒(epstein-barr virus, EBV)感染. 病毒感染可 以是原发的感染或潜在感染的激活, 临床表现可以

英文版见: Zhang Y W, Zhao H W, He X, et al. Effects of Epstein-Barr virus infection on the development of multiple myeloma after liver transplantation. Sci China Life Sci, 2012, 55: in press 
从无症状的隐性感染到严重的懪发性感染 ${ }^{[2]}$. 患者通 常可以自行康复, 但有些并发症如肝炎, 传染性单核 细胞增多症, 移植后淋巴增生症等往往会进一步发 展. 由于肝移植术后的免疫抑制, $\mathrm{T}$ 细胞的功能受限, 使 B 细胞呈单克隆性增生并导致肿瘤发生 ${ }^{[2,3]}$.

$\mathrm{EBV}$ 是一种 $\gamma$ 亚科疮疹病毒, 它和人类很多恶性 疾病有关, 特别是上皮和淋巴起源的肿瘤. EBV 在体 外能感染正常静止的 B 细胞, 使它们变成永生化的 淋巴细胞系(LCL). LCLs 表达的病毒抗原中包括 EBV 核抗原(EBNA1 6)、潜伏性膜蛋白(LMP1 和 LMP2) 以及 $\mathrm{EBV}$ 编码的 RNAs(EBERs) ${ }^{[4]}$. 其中, LMP1 被认 为在 $B$ 细胞的转化过程中起着关键作用. 但是, 有研 究表明, 仅依靠 LMP1 的表达不足以维持 B 细胞的体 外增殖, 它还需要第二信号的辅助 ${ }^{[5]}$. CD40 在下游信 号传导方面与 LMP1 有共同通路, 被认为可能是主要 的 LMP1 信号传导辅助分子.

人 CD40 分子是 I 型跨膜糖蛋白, 属于肿瘤坏死 因子 $(\mathrm{TNF})$ 受体超家族成员, 它可以表达在不同分化 阶段的 B 淋巴细胞上 ${ }^{[6]}$, 也包括一些 B 淋巴细胞起源 的恶性肿瘤如多发性骨髓瘤细胞上。它的配体 CD40L 属于 TNF 超家族成员, 主要瞬时表达于活化 的 $\mathrm{CD}^{+} \mathrm{T}$ 细胞上. 有研究发现, CD40L 也可以表达 在正常或肿瘤 $\mathrm{B}$ 细胞上 ${ }^{[7,8]}$, 而异常的 CD40-CD40L 的相互作用或异位的 CD40L 表达能使本应该调亡和 被清除的肿瘤细胞存活下来 ${ }^{[9]}$.

多发性骨髓瘤是源于 B 细胞分化终末期的浆细 胞的恶性肿瘤 ${ }^{[10]}$, 肿瘤细胞主要定居于骨髓, 与骨髓 基质细胞关系密切, 在骨髓中大量增生, 可占到骨髓 内细胞总数的 15\% 90\%. 骨髓瘤的确切致病因素到 目前为止不甚清楚, 可能与生发中心瞬时疮疹病毒 感染有关 ${ }^{[11]} . \mathrm{EB}$ 病毒作为疮疹病毒的一种, 近几年 备受关注.

本实验首先通过流式细胞仪检测 10 例肝脏移植 术后感染 EBV 患者并发多发性骨髓瘤新鲜骨髓标本 中 CD40 和 CD40L 共表达情况, 观察共表达在 B 细 胞起源的恶性肿瘤的普遍性. 然后着重研究骨髓瘤 细胞株 RPMI8226 在 EB 病毒感染后, 其表型和细胞 生物学行为的改变, 尤其是 CD 40 和 CD $40 \mathrm{~L}$ 的共表达 情况. 研究 CD40-CD40L 在肿瘤细胞逃逸 GC 机制所 发挥的作用, 来探讨浆细胞逃脱调亡演变成恶性骨 髓瘤细胞的可能机制及 $\mathrm{EB}$ 病毒感染对肝脏移植术后 并发骨髓瘤细胞表型和生物学的影响.

\section{1 材料与方法}

\section{1 试剂、细胞株和骨髓标本}

鼠抗人 CD40 单克隆抗体(5C11,3G3-FITC)、鼠 抗人 CD40L 单克隆抗体(4F1)、LMP1 单克隆抗体、 EBNA-2 多克隆抗体、CXCR4 单克隆抗体均购自美 国 Santa Gruz 生物技术公司; FITC-二抗、Cy-3 二抗、 Ig 同型对照均购自法国 Immunotech 公司; RNA 抽提 试剂盒、Western blotting 显色试剂盒购自中国上海华 顺公司; RT-PCR 试剂盒购自日本 TaKaRa 公司; Annexin-FITC 购自德国 Boehringer Mannhei 公司; Transwell 板购自德国 Corning Costar 公司; 趋化因子 SDF-1 $\alpha$ 购自美国 BD 公司. 10 例肝移植术后有 EBV 感染病史并发骨髓瘤患者的骨䯣瘤新鲜标本由中国 活体肝脏研究所提供; 多发性骨髓瘤细胞株 RPMI 8226, EB 病毒产生细胞株 B95-8 和恶性淋巴瘤细胞 株 Raji, Daudi 均购自美国 ATCC 公司; 培养基 RPMI1640 和 FCS 购自美国 GeBico 公司. 所有细胞 株均用德国 Boehringer Mannhei 公司的酶标试剂盒检 测, 没有支原体污染.

新鲜骨髓标本取自 10 例肝脏移植术后并发多发性 骨髓瘤患者, 患者分别于移植后第 9 29 个月内出现骨 䯣瘤临床表现, 经临床诊断(国际骨髓瘤工作组, 2003) 为多发性骨髓瘤, 均经酶联免疫吸附测定法证实 $\mathrm{EBV}$ 感染, 血清 EBV-IgM 抗体阳性, 经抗 EBV 治疗后, 均 治愈. 病例采集符合“赫尔辛基宣言”，并在南京医科大 学附属江苏省肿瘤医院伦理委员及每位患者同意的前 提下进行. 男性 7 例, 女性 3 例; 年龄 14 45 岁, 平均 27 岁. 10 例患者, 均为原发性肝癌, 肝炎病毒标志物 (B/C), $n=7 / 3$; HBV DNA(+/-), $n=4 / 6$; 肝功能 Child 分级 $(\mathrm{A} / \mathrm{B} / \mathrm{C}), n=8 / 2 / 0$; 甲胎蛋白 $(+/-), n=8 / 2$. 一位患者术前 行动脉栓塞化疗. 肿瘤侵犯门静脉主干, $n=0$. 侵犯门静 脉左右属支, $n=4$. 侵犯肝静脉, 腔静脉, $n=0$. 无瘤侵犯, $n=6$. 所有患者术前 EBV-IgM 抗体均为阴性.

7 例应用环狍素 A 联合泼尼松, 3 例应用他克莫 司联合泼尼松, 3 例患者加用霉酚酸酯. 10 例患者在 诊断为 $E B V$ 感染前均有激素(100 200 mg 甲泼尼龙 琥珀酸钠)冲击治疗史. HBSAg 阳性患者口服拉米呋 定. 7 例患者分别于术后 $9,11,12,14,19$ 和 28 天出 现干咳、发热、呼吸困难, 胸部 X 线显示双肺多片 状阴影, 呈间质性肺炎表现. 3 例患者分别于术后 23 , 25 和 27 天出现顽固性腹泻伴有肌肉酸痛、关节痛、 
恶心、呕吐. 10 例患者均有发热; 4 例咽分泌物增多; 7 例淋巴结(3 例颈部淋巴结、4 例下领骨周围淋巴结) 肿大; 2 例肝脏肿大; 3 例脾脏肿大; 4 例肝脏和脾脏 均肿大; 3 例出现皮疹; 3 例罹患支气管炎和支气管 肺炎.

\section{2 流式细胞仪检测 CD40 和 CD40L 在骨髓瘤细 胞中的表达}

按照标准 Ficoll-Histopaque 过程从 10 例 EB 病毒 感染的肝移植术后患者收集的新鲜的多发性骨髓瘤 细胞. 按操作手册, 将 $3 \mu \mathrm{L}$ 异硫氰酸荧光素(FITC)标 记的 CD40 单抗以及藻红蛋白标记的 CD40L或 $\mathrm{CD} 138$ 单抗分别加入细胞悬液 $100 \mu \mathrm{L}\left(1 \times 10^{6}\right.$ 个细胞 $)$. 在室温中避光培养 $30 \mathrm{~min}$ 后, 用 $1 \mathrm{~mL}$ 自制流式细胞 仪专用溶血素溶液(溶血素: 去离子水 $=1: 9$ )避光处理 染色样品 $10 \mathrm{~min}$. 样品用 PBS 液冲洗, 在室温下 $450 \times g$ 离心 $5 \mathrm{~min}$. 细胞在避光 $300 \mu \mathrm{L}$ 聚甲醛溶液室 温下固定 $15 \mathrm{~min}$, 或 $4^{\circ} \mathrm{C}$ 过夜, 然后用 FACSFlow 溶 液(美国 BD 公司)冲洗. 细胞颗粒悬浮在 FACSFlow 溶液中, 并用流式细胞仪(美国 $\mathrm{BD}$ 公司)分析.

\section{$1.3 \mathrm{~EB}$ 病毒子的制备}

根据 $\mathrm{Oh}$ 等人 ${ }^{[12]}$ 的实验方法, 在指数生长的 $\mathrm{B} 95-8$ 细胞 $\left(5 \times 10^{5} / \mathrm{mL}\right)$ 中, 用 $100 \mathrm{nmol} / \mathrm{L}$ 的 $12-\mathrm{O}-$ 一四烷酰 佛波醋酸酯-13(12-O-tetradecanoylphorbol-13-acetate, TPA)刺激 $2 \mathrm{~h}$, 然后用 HEPES 缓冲液洗 3 遍以移去溶 液中的 TPA, 将细胞培养 $48 \mathrm{~h}$ 后, $850 \times g$ 离心 $3 \mathrm{~min}$ 收获上清. 再将上清通过 $0.22 \mu \mathrm{m}$ 滤器过滤, 用离心 管分装 $1 \mathrm{~mL} /$ 支，存储于 $4^{\circ} \mathrm{C}$ 以备用.

\subsection{EB 病毒的感染}

通过富集病毒和延长感染时间, 使 RPMI8226 细 胞获得有效感染. 取对数生长期的 RPMI8226 细胞 (总数 $3 \times 10^{6}$ ) 离心 $5 \mathrm{~min}$, 弃上清. 再取 $3 \mathrm{~mL}$ 已制备好 的病毒与细胞共培养 $16 \mathrm{~h}$, 然后用培养基洗 2 遍移去 病毒, 再加入 $10 \%$ FCS 的 RPMI1640 继续培养. 对照 组使用紫外灯 $(15 \mathrm{~W}, 60 \mathrm{~cm}, 60 \mathrm{~min})$ 灭活的 $\mathrm{EB}$ 病毒 感染 RPMI 8226 细胞.

\section{5 免疫苂光抗体和细胞表型分析}

感染后 $48 \mathrm{~h}$, 实验分 2 组: 对照组 RPMI8226 细 胞未经 EB 病毒感染, 在 10\%FCS 的 RPMI1640 生长;
实验组 RPMI8226 细胞先经过 EB 病毒感染, 再恢复 到 $10 \%$ 胎牛血清(FCS)的 RPMI1640 继续生长. 将各 组细胞调整到 $5 \times 10^{6} / \mathrm{mL}$, 取 $100 \mu \mathrm{L}$ 分别加入 $1 \mu \mathrm{g}$ 的 CD40 单抗(5C11)、1 $1 \mu \mathrm{g}$ 的 CD40L 单抗(4F1)、1 $\mu \mathrm{g}$ 的 LMP1 单抗, $4^{\circ} \mathrm{C}$ 孵育 $30 \mathrm{~min}, \mathrm{PBS}$ 洗 2 次. 再分别 和 FITC 标记的二抗 $4^{\circ} \mathrm{C}$ 孵育 $30 \mathrm{~min}, \mathrm{PBS}$ 洗 2 次, 悬 浮于 $0.5 \mathrm{~mL}$ 的 PBS 中, 流式细胞仪分析. 每组实验 重复 3 次, 结果取均值.

\section{6 反转录酶-聚合酶链反应(RT-PCR)}

感染后 $24 \mathrm{~h}$, 调整各组细胞总数至 $5 \times 10^{6}$. 取经 紫外线灭活的 EB 病毒感染 RPMI 8226 细胞作对照组. 按照 RNA 抽提试剂盒说明书, 抽取 RNA. 逆转录成 cDNA 进行 PCR: $94^{\circ} \mathrm{C} 30 \mathrm{~s}, 56^{\circ} \mathrm{C} 30 \mathrm{~s}, 72^{\circ} \mathrm{C} 45 \mathrm{~s}, 34$ 个 循环. CD40L 胞外段引物序列为, 上游引物: 5'-GCCGAATTCGAAAACAGCTTT-3', 下游引物: 5'-GCCTCTAGAAATCAGAGTTTGAGT-3'(法国 Immunotech 公司合成); $1 \%$ 琼脂糖凝胶电泳. $\beta$-actin 引物序列为, 上游引物: 5'-TCATGAAGTGTGACGTGGACATC-3', 下游引物： 5'-TCTAGTTCTAGTAACGAGGAGGAC$3^{\prime}$. $\beta$-actin 片段扩增操作如下: $94^{\circ} \mathrm{C} 30$ 秒, $53{ }^{\circ} \mathrm{C} 30 \mathrm{~s}$, $72^{\circ} \mathrm{C} 30 \mathrm{~s}, 30$ 个循环; 最后在 $72^{\circ} \mathrm{C}$ 孵育 $3 \mathrm{~min}$ 延伸. 对获得的 PCR 产物行 $1 \%$ 琼脂糖凝胶电泳.

\subsection{Western blotting}

将两组各 $10^{6}$ 个 RPMI8226 细胞用 PBS 洗 2 次. 取经紫外线灭活的 EB 病毒感染 RPMI 8226 细胞作对 照组. 将细胞重悬于冷的 RIPA 缓冲液 $(1 \times \mathrm{PBS}$, $1 \% \mathrm{NP}-40,0.5 \%$ 脱氧胆酸钠, $0.1 \% \mathrm{SDS})$ 中, 再加蛋白 酶抑制剂(10 mg/mL PMSF, $30 \mu \mathrm{g} / \mathrm{mL}$ 抑肽酶, 100 $\mathrm{mmol} / \mathrm{L} \mathrm{Na}_{3} \mathrm{VO}_{4}$ ). 在冰上放置 $30 \mathrm{~min}$, 并用 $1 \mathrm{~mL}$ 针 筒来回抽吸数次, $4^{\circ} \mathrm{C}, 1000 \times g$ 离心 $20 \mathrm{~min}$. 上清用于 SDS-PAGE 电泳, 并转移到硝酸纤维膜上, 用 $1 \%$ 的 封闭液封闭 $1 \mathrm{~h}$, 加入 LMP1 单抗或 EBNA-2 多抗孵 育 $1 \mathrm{~h}$, 用 TBST洗去未结合的抗体, 加入碱性磷酸酶 标记的羊抗鼠二抗, 孵育 $1 \mathrm{~h}$, 用 TBST 洗 3 次, 再加 入底物显色. 具体实验操作可参考试剂盒说明书(美 国 $\mathrm{BD}$ 公司). 通过细胞膜上的 3-磷酸甘油醛脱氢酶 $(\mathrm{GAPDH})$ 染色, 验证等价的蛋白荷载和传输效率.

\section{8 细胞周期检测和调亡分析}

周期检测. 将细胞密度调至 $5 \times 10^{6}$ 个 $/ \mathrm{mL}$, 取出 
$100 \mu \mathrm{L}$ 与 $500 \mu \mathrm{L}$ 碘化丙啶 $(\mathrm{PI})$ 缓冲液 $\left(450 \mu \mathrm{L} \mathrm{H} \mathrm{H}_{2} 0\right.$, $0.05 \mathrm{~mol} / \mathrm{L}$ 柠檬酸钠, $50 \mu \mathrm{g}$ PI, $0.05 \%$ Triton 100,100 $\mu \mathrm{g} / \mathrm{mL}$ RNase)混合, 避光 $4^{\circ} \mathrm{C}$ 放置 $30 \mathrm{~min}$, 流式细胞 仪分析.

调亡分析. 调亡细胞用 PI 和 Annexin- $V$ 双标记 检测. $5 \times 10^{5}$ 个细胞用 PBS 洗 2 次, 把细胞重悬于 500 $\mu \mathrm{L}$ PI 冷的缓冲液中, 在缓冲液中加入 $5 \mu \mathrm{L}$ FITCAnnexin- $\mathrm{V}$ 和 $5 \mu \mathrm{L} \mathrm{PI}$, 避光 $4^{\circ} \mathrm{C}$ 㽟育 $10 \mathrm{~min}$, 流式细 胞仪分析. 详细的实验操作参阅试剂盒说明书(法国 Immunotech 公司).

\section{9 细胞向趋化因子 SDF-1 $\alpha$ 迁徙能力检测}

选择孔径为 $8 \mu \mathrm{m}$ 的 Transwell 板, 分为 4 组, $\mathrm{A}$ 组: EBV(-)RPMI8226+SDF-1 $\alpha(-)$ 10\% FCS RPMI 1640; B 组: EBV(+)RPMI8226+SDF-1 $\alpha(-)$ 10\% FCS RPMI1640; C 组: EBV(-)RPMI 8226+SDF-1 $\alpha(+)$ 10\% FCS RPMI 1640; D 组: EBV(+)RPMI 8226+SDF- $1 \alpha(+) 10 \%$ FCS RPMI 1640. 上室加不同组细胞 $10^{5}$ 个 $/ 100 \mu \mathrm{L}$, 下室 加含有 $200 \mathrm{ng} / \mathrm{mL}$ 趋化因子 SDF-1 $\alpha$ 的 $600 \mu \mathrm{L} 10 \%$ FCS RPMI 1640 培养基. $37^{\circ} \mathrm{C}$ 温育 $4 \mathrm{~h}$ 后, 收集下室细 胞上流式细胞仪计数, 观测各组细胞的迁徙能力.

\subsection{0 激光共聚焦显微镜分析}

调整细胞浓度至 $1 \times 10^{5} / \mathrm{mL}, \mathrm{PBS}$ 洗涤后, 加 100 $\mu \mathrm{L}$ 固定液, 重悬细胞, 室温保持 $15 \sim 30 \mathrm{~min}$. 加 $1 \mathrm{~mL}$ $\mathrm{PBS} / \mathrm{BSA}$ (含 $1 \mu \mathrm{g} / \mathrm{mL} \mathrm{BSA}$ 的 PBS), 重悬, 离心, 洗 涤 2 遍. 分为 2 组: 一组加 $100 \mu \mathrm{L}$ 反应液 I (含 10 $\mu \mathrm{g} / \mathrm{mL}$ CD40 多克隆抗体和 $10 \mu \mathrm{g} / \mathrm{mL}$ LMP1 单克隆抗 体的 PBS/BSA), 重悬细胞, 室温保持 30 60 min. 加 $1 \mathrm{~mL}$ PBS/BSA, 重悬, 离心, 洗涤 2 遍. 加 $100 \mu \mathrm{L}$ 反应液 II (含 $20 \mu \mathrm{g} / \mathrm{mL}$ Cy5-羊抗兔 $\operatorname{IgG}$ 和 $20 \mu \mathrm{g} / \mathrm{mL}$ Cy-3 羊抗小鼠 $\operatorname{IgG}$ 的 PBS/BSA), 重悬细胞, 室温保 持 30 45 min, 洗涤; 另一组加 $100 \mu \mathrm{L}$ 反应液 I (含 $10 \mu \mathrm{g} / \mathrm{mL}$ CD $40 \mathrm{~L}$ 单克隆抗体 $4 \mathrm{~F} 1$ 的 PBS/BSA), 重悬

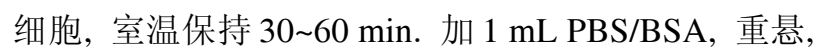
离心, 洗涤 2 遍. 加 $100 \mu \mathrm{L}$ 反应液 II (含 $20 \mu \mathrm{g} / \mathrm{mL}$ Cy-3 羊抗小鼠 IgG 的 PBS/BSA), 重悬细胞, 室温保 持 30 45 min, 洗涤. 再加入 $100 \mu \mathrm{L}$ 反应液 III(含 10 $\mu \mathrm{g} / \mathrm{mL} 3 \mathrm{G} 3$-FITC 的 PBS/BSA), 重悬细胞, 室温保持 30 45 min, 洗涤. 两组细胞 PBS 洗涤 1 遍, 加 $20 \mu \mathrm{L}$ 封片液, 重室细胞. 各取 $10 \mu \mathrm{L}$ 上述含有细胞的封片 液, 滴加在载玻片上, 加上盖玻片后在共聚焦显微镜
(美国 Bio-Rad 公司)下观察.

\subsection{1 细胞增殖}

将 Daudi 细胞用 10\%FCS RPMI1640 培养基洗一 次, 用 $10 \%$ FCS RPMI1640 调节细胞浓度至 $1 \times 10^{5}$ 个/ $\mathrm{mL}$. 取 $100 \mu \mathrm{L}$ 细胞悬液加到 96 孔板中, 分别加入 $\mathrm{IgG}$ 和 CD $40 \mathrm{mAb}$ 各 $0,5,10$ 和 $20 \mu \mathrm{g} / \mathrm{mL}$. 放入 $37^{\circ} \mathrm{C}$ 、 $5 \% \mathrm{CO}_{2}$ 培养箱中, 培养 $24 \mathrm{~h}$, 每孔掺入 ${ }^{3} \mathrm{H}-\mathrm{TdR} 3$. $7 \times 10^{4} \mathrm{~Bq}$, 继续培养 $16 \mathrm{~h}$ 终止, 收获细胞, 用闪光仪 测定 $\mathrm{cpm}$ 值, 每组实验重复 3 次, 结果取均值.

\subsection{2 细胞调亡检测}

将 Daudi 或 Raji 细胞分别设置 4 组: (1) 对照组,

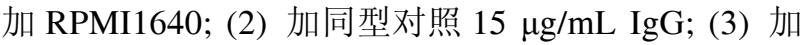
$15 \mu \mathrm{g} / \mathrm{mL}$ CD40mAb; (4) 加 $15 \mu \mathrm{g} / \mathrm{mL}$ CD40LmAb. 6 孔板中细胞培养 $24 \mathrm{~h}$, 调亡细胞用 PI 和 Annexin- $V$ 双 标记检测. $5 \times 10^{5}$ 细胞用 PBS 洗 3 次, 把细胞重悬于 $500 \mu \mathrm{L}$ PI 冷的缓冲液当中, 在缓冲液中加入 $5 \mu \mathrm{L}$ FITC-Annexin- $\mathrm{V}$ 和 $5 \mu \mathrm{L}$ PI，避光 $4^{\circ} \mathrm{C}$ 捊育 $10 \mathrm{~min}$ ，流 式细胞仪分析.

\subsection{3 统计学处理}

使用 SPSS11.0 软件进行 $t$ 检验分析数据, 实验数 据以 $\bar{x} \pm \mathrm{SD}$ 表示, $P<0.05$ 为差别有统计学意义.

\section{2 结果}

\subsection{0 例骨髓瘤新鲜标本 CD40 和 CD40L 共表达 情况}

CD138 是骨髓瘤的标记分子，通过检测 CD138 和 CD40 或 CD138 和 CD40L 双标记, 来检测骨髓瘤 细胞 CD40 和 CD40L 的表达情况. 10 例标本中 CD40 和 CD40L 都有不同程度的表达. 其中 5,8,9 和 10 号 标本 CD40 和 CD40L 都有明显共表达 $(P>0.05)$ (图 1), 特别是 5 号标本 $\mathrm{CD} 40$ 和 $\mathrm{CD} 40 \mathrm{~L}$ 呈高度共表达.

\section{$2.2 \mathrm{~EB}$ 病毒能感染 RPMI8226 细胞并在 RNA 水} 平及蛋白水平 CD40L 分子表达

RPMI 8226 细胞在体外与含有 EB 病毒颗粒的上 清共培养后, 经免疫苂光抗体标记和流式细胞仪检 测表明, 感染细胞表面开始表达 LMP1(图 2A). 结果 表明，约 $95 \%$ 的 RPMI 8226 细胞感染 EB 病毒. 


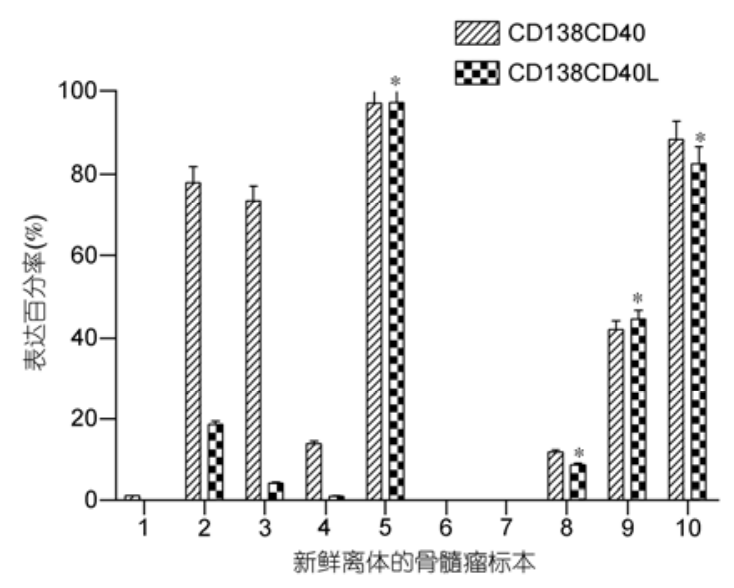

图 110 例骨髓瘤新鲜标本 CD40 和 CD40L 共表达情况 $5,8,9$ 和 10 号标本 CD40 和 CD $40 \mathrm{~L}$ 都有明显共表达(*: P>0.05), 特 别是 5 号标本 CD40 和 CD40L 呈高度共表达
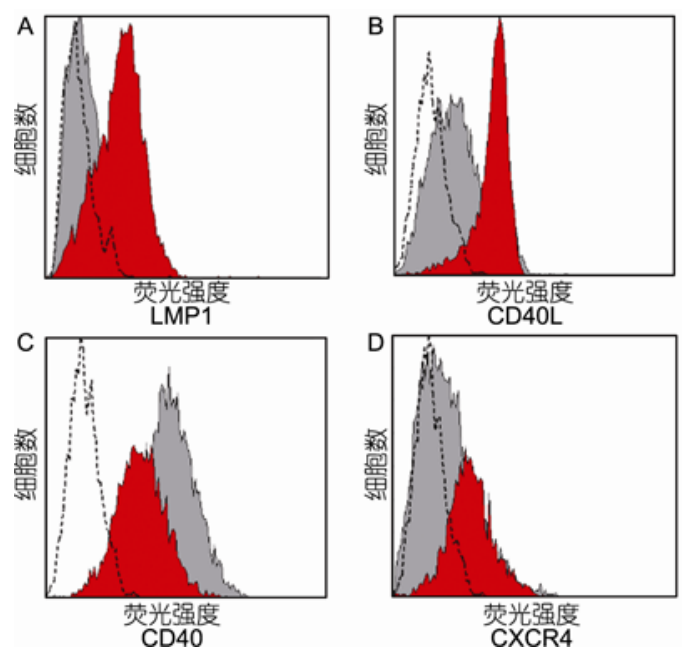

图 2 流式细胞术测定表型

感染后 $48 \mathrm{~h}$, 测定细胞表面标志物的表达情况: 灰色区域示未感染 细胞; 红色区域示感染 EB 病毒的 RPMI 8226 细胞; 虚线围成区域 示同型对照细胞. 细胞膜表面的 LMP1, CD40L 和 CXCR4 表达增多, CD40 表达减少

Western blotting 分析的结果也发现细胞感染后有 LMP1 和 EBNA-2 的表达(图 3A). RT-PCR 分析显示, RPMI 8226 细胞在感染后 $24 \mathrm{~h}$ 就有 CD40L 上调表达 (图 3B), 而在感染后 $48 \mathrm{~h}$, 流式细胞仪检测也证实膜 表面 CD40L 呈上调表达(图 2B), 然而 CD40 却呈现 下调性表达(图 2C).

\subsection{EB 病毒感染赋予 RPMI8226 细胞感染后抗调 亡的能力}

$\mathrm{EB}$ 病毒感染 8226 细胞 $24 \mathrm{~h}$ 后，细胞周期没有明

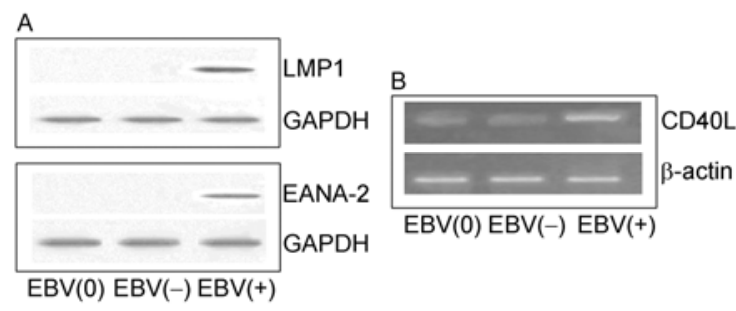

图 3 生化检测结果

A: 感染后 $24 \mathrm{~h}$ Western blotting 的检测 LMP1 和 EBNA-2 表达. $\operatorname{EBV}(0)$ : 感染紫外线灭活 EB 病毒的 RPMI 8226 细胞; EBV(-): 未 感染 EB 病毒的 RPMI 8226 细胞; EBV(+): 感染 EB 病毒的 RPMI 8226 细胞. 上图示 LMP1 表达; 下图示 EBNA-2 表达. B: 感染后 24 h RT-PCR 检测 CD40L mRNA. EBV(0): 感染紫外线灭活 EB 病毒的 RPMI 8226 细胞; EBV(-): 未感染 EB 病毒的 RPMI 8226 细胞 (CD40L mRNA 正常表达); EBV(+): 感染(CD40L mRNA 上调表达)

显变化, 但在 G1 期前有小调亡峰出现(图 4B, 箭头所 示); 感染后 $48 \mathrm{~h}$, 也是 CD40L 上调表达时, 调亡峰 消失(图 4C). 通过 Annexin-V-PI 双标记检测感染细胞 调亡率发现，未感染 RPMI 8226 细胞调亡率为 $2.35 \% \pm$ $0.45 \%$ ，感染后 $24 \mathrm{~h}$ 调亡率上升到 $12.30 \% \pm 2.10 \%$ ，然 而在感染后 $48 \mathrm{~h}$, 也是 CD $40 \mathrm{~L}$ 上调表达时, 调亡率 下调到 $3.92 \% \pm 0.42 \%$ (图 5).

\subsection{EB 病毒感染后上调 8226 细胞表达功能性趋化 因子受体 CXCR4}

流式细胞仪检测表明, RPIM8226 细胞在感染后 $48 \mathrm{~h}$ 表达趋化因子受体 CXCR4(图 2D). 而且感染细 胞在趋化因子 SDF-1 $\alpha$ 趋化作用下，显著地增强了迁 移率 $\left({ }^{*} P<0.05\right)$ (图 6).

\section{$2.5 \mathrm{CD40}, \mathrm{CD} 40 \mathrm{~L}$ 和 LMP1 分子在感染细胞的共 聚集}

用直标抗体 3G3-FITC 标记细胞表面 CD40 分子, 显示为绿荧光; 用间标抗体 4F1 或 anti-LMP1 标记细 胞表面 CD40L 分子, 再联合 Cy3 二抗, 显示为红荧 光; 如果 2 个分子在细胞表面共聚集, 则显示为黄苂 光. 共聚焦显微镜分析显示, 感染后的 RPMI8226 细 胞形态变的不规则, 表达的 CD40 和 CD40L 或 EBV 病毒蛋白 LMP1 能在细胞表面共聚集(黄荧光)(图 7). 由此推测, EBV 病毒蛋白 LMP1 的整合可能参与 CD40 信号的传导. 

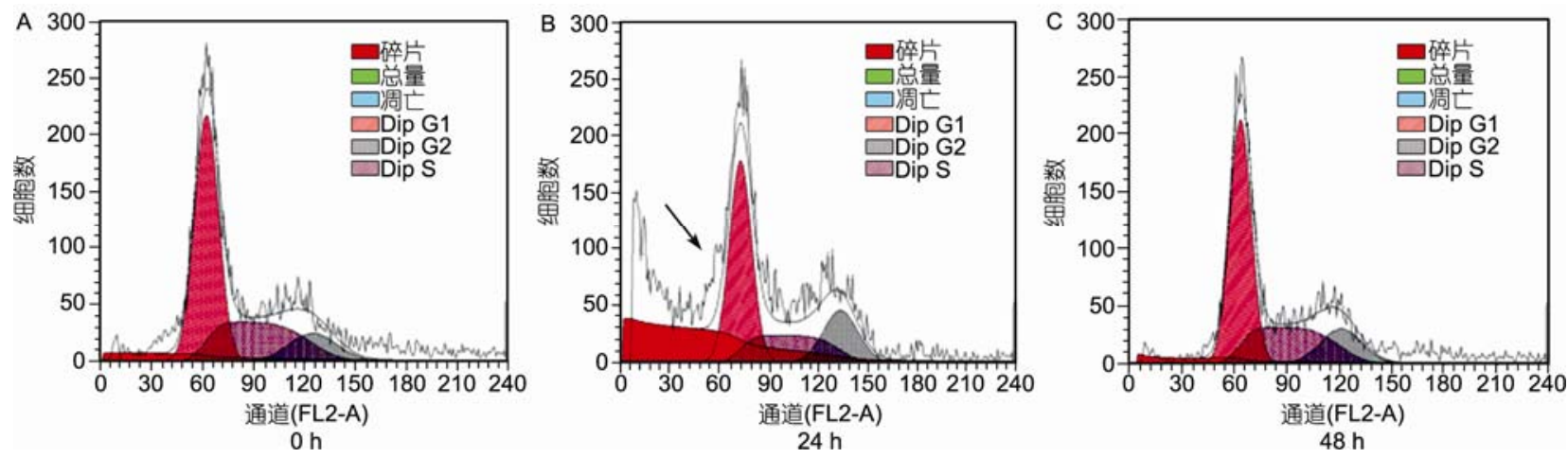

图 4 流式细胞术检测 PI 染色的感染 EB 病毒的 RPMI 8226 细胞周期

A: 感染后 $0 \mathrm{~h} ; \mathrm{B}$ : 感染后 $24 \mathrm{~h}$, 细胞周期没有明显变化, 但在 $\mathrm{G} 1$ 期前有小调亡峰出现(箭头所示); C: 感染后 $48 \mathrm{~h}$, 即 CD $40 \mathrm{~L}$ 上调表达时, 调 亡峰消失

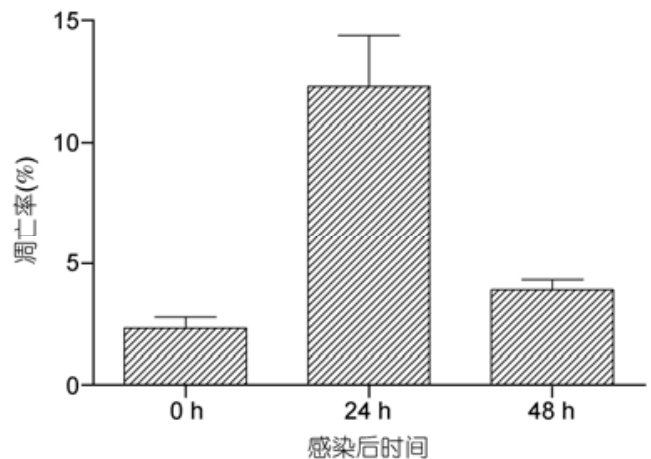

图 $5 \mathrm{~EB}$ 病毒感染后 RPMI 8226 细胞的调亡率 未感染 $\mathrm{EB}$ 病毒时 RPMI 8226 细胞的调亡率为 $2.35 \% \pm 0.45 \%$; EB 病 毒感染 $24 \mathrm{~h}$ 后 RPMI 8226 细胞的调亡率为 $12.30 \% \pm 2.10 \%$ (与未感染 及感染 $48 \mathrm{~h}$ 后相比, $P<0.05$ ); $\mathrm{EB}$ 病毒感染 $48 \mathrm{~h}$ 后, CD $40 \mathrm{~L}$ 上调表达, 此时 RPMI 8226 细胞的调亡率为 $3.92 \% \pm 0.42 \%$

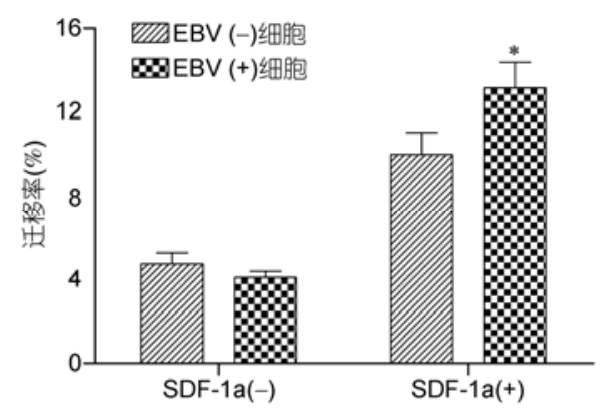

图 $6 \mathrm{~EB}$ 病毒感染后 RPMI 8226 细胞的迁移率

未加入趋化因子 SDF- $1 \alpha$ 时, 未感染 EB 病毒的 RPMI 8226 细胞的调 亡率为 $4.79 \% \pm 0.52 \%$, 感染 EB 病毒的 RPMI 8226 细胞的调亡率为 $4.14 \% \pm 0.31 \%$; 加入 SDF-1 $\alpha$ 后, 未感染 EB 病毒的 RPMI 8226 细胞 的调亡率为 $10.04 \% \pm 1.03 \%$, 感染 EB 病毒的 RPMI 8226 细胞的调亡 率为 $13.18 \% \pm 1.21 \%$ (与未加入趋化因子 SDF-1 $\alpha$ 的感染 EB 病毒的 RPMI 8226 细胞相比, $* P<0.05)$

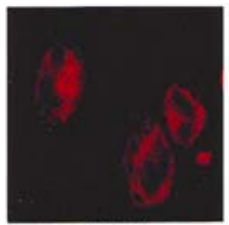

LMP1

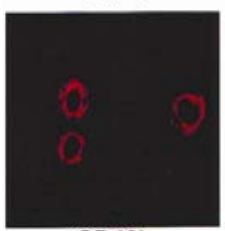

CD40L

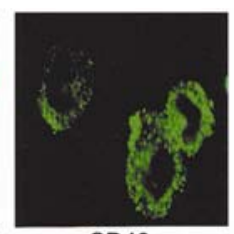

CD40

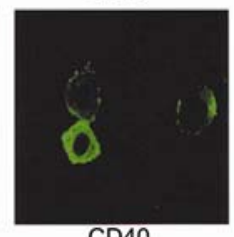

CD40

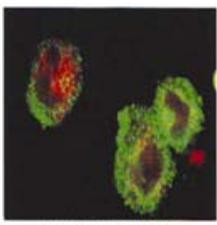

LMP1/CD40

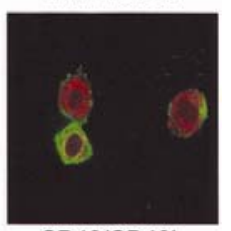

CD40/CD40L
图 7 共聚焦显微镜分析 CD40, CD40L 和 LMP1 分子在感 染细胞的共聚集

CD40/CD40L 和 LMP1/CD40 在细胞表面共聚集, 共聚集区域呈现 黄色苂光

\subsection{CD40-CD40L 信号对 Daudi 细胞增殖的影响}

用 3 种不同的处理方式: 同型 IgG、CD40 单抗、 CD40L 单抗处理 Daudi 细胞, 观察对细胞增殖的影响. 实验结果显示, 相对于同型对照组, CD40 单抗或 CD40L 单抗都能抑制细胞增殖, 而且这种增殖抑制 呈剂量依赖性, 实验还发现, CD40L 单抗对 Daudi 的 增殖抑制作用强于 CD40 单抗(图 8).

\subsection{CD40-CD40L 信号对 Raji 细胞调亡的影响}

用 3 种不同的处理方式: 同型 $\mathrm{IgG}, \mathrm{CD} 40$ 单抗及 CD40L 单抗处理 Raji 细胞, 观察对细胞调亡的影响. 实验结果显示, 第一象限调亡细胞比例有不同程度 的改变. 进一步分析发现, 无论是 CD40 单抗还是 CD40L 单抗都能使 Raji 细胞调亡率增高, 而只有 
CD40L 单抗能使 Daudi 细胞调亡率明显增高, CD40 单抗则作用不明显(图 8).

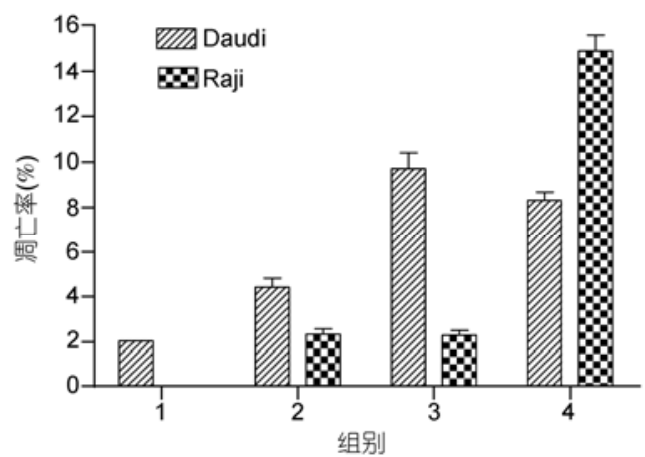

图 8 不同处理下 Daudi 或 Raji 的调亡率

1: RPMI 1640 培养基; $2: 15 \mu \mathrm{g} / \mathrm{mL} \mathrm{gG}$ 作为同型对照; $3: 15 \mu \mathrm{g} / \mathrm{mL}$ CD40 单抗; $4: 15 \mu \mathrm{g} / \mathrm{mL}$ CD 40L 单抗. 6 孔板中细胞培养 $24 \mathrm{~h} . \mathrm{CD} 40$ 单抗和 $\mathrm{CD} 40 \mathrm{~L}$ 单抗都增加了 Raji 细胞的调亡率; 只有 $\mathrm{CD} 40 \mathrm{~L}$ 单抗 增加了 Daudi 细胞的调亡率, CD40 单抗对 Daudi 细胞的调亡率无明 显影响

\section{3 讨论}

CD40 是分子量 45 50 kD 的膜分子, 属于 TNFR 超家族成员. CD40 与 EBV 转化后的 B 细胞所表达的 病毒抗原 LMP1 在信号传导方面有很多的相似性. 并 且当 CD40 配基化时, 细胞表面 CD40 和 LMP1 分子 可被募集到脂筏组成信号传导复合体传递信号. CD40 的配体 CD40L, 又称 CD154, 是分子量为 $39 \mathrm{kD}$ 的膜分子，属于 $\mathrm{TNF}$ 超家族成员. $\mathrm{CD} 40 \mathrm{~L}$ 主要以三聚 体的形式瞬时表达在抗原活化的 $\mathrm{CD}^{+} \mathrm{T}$ 细胞表面, 也可以瞬时表达在生发中心的 B 细胞和异位表达在 一些 $\mathrm{B}$ 细胞系来源的肿瘤细胞上. B 细胞的成熟需要 通过在生发中心与抗原活化的瞬时表达的 $\mathrm{CD}^{+} \mathrm{T}$ 细 胞结合，促使 B 细胞表面的 CD40 配基化，才能使高 抗原亲和力的、非自身免疫性的 B 细胞存活、增殖、 分化. 生发中心 B-T 细胞间 CD40-CD40L 的相互作用 能够使 $\mathrm{B}$ 细胞免于调亡. 但是, 生发中心中 $\mathrm{T}$ 细胞数 量很少, 自身正常 B 细胞表达 CD40L 也是暂时的, 这种严格的笚选机制只能使一部分功能性 B 细胞存 活, 而未能和 $\mathrm{T}$ 细胞结合发生配机化的恶性细胞和或 自身免疫细胞则被清除. 然而, 许多肿瘤细胞能逃脱 生发中心的篎选机制, 最终存活下来得到扩增. 近来 有研究发现, 受体和配体可以在相同的细胞共表达, 尤其是在恶性细胞 ${ }^{[13]}$. 一些致癌因素, 如病毒感染可
以导致异位表达或表达上调，并最终使恶性细胞逃 避调亡或免疫细胞的攻击. 恶性细胞中受体和配体 共表达的具体机制, 值得进一步研究.

很多 B 细胞起源的恶性肿瘤都出现了 CD40 和 CD40L 的异位表达. 而且, 很多致癌因素如病毒感 染在转化细胞的过程中，也利用了 CD40-CD40L 这 条途径 ${ }^{[14]}$. 肿瘤 B 细胞在什么方面不同于正常 B 细 胞, 从而在生发中心中能够逃脱严格的篮选机制, 存 活下来并得到无限扩增? 是否通过自身提供 CD40L, 或上调 CD40L，使得在生发中心无法通过 $\mathrm{T}$ 细胞依赖 途径存活的肿瘤细胞存活下来? 因为 CD40-CD40L 与 细胞生长增殖密切相关, 研究肿瘤细胞上这对分子, 或在特定条件下如病毒感染，观察这对分子表达的 前后变化及相应的生物学行为改变, 为临床探索肿 瘤的发生发展提供重要信息.

本研究应用有效制备 EB 病毒的方法, 发现 EB 病毒作为一种 $\gamma$-亚科疮疹病毒, 能在体外感染 B 细胞 来源的恶性浆细胞，即多发性骨髓瘤细胞株 RPMI8226 细胞. 通过流式细胞仪和 Western blotting 的检测, 证明感染后细胞在蛋白水平有 LMP1 和 EBNA-2 这两个 EB 病毒感染后标记分子的表达. 通 过流式细胞仪和 RT-PCR, 分别从蛋白水平和 RNA 水平显示了 $\mathrm{CD} 40 \mathrm{~L}$ 表达在 $\mathrm{EBV}$ 感染后呈上调. 有文 献报道 ${ }^{[15,16]}$, LMP1 在 EB 病毒转化 B 细胞中起着关 键作用。它在下游信号传导途径和募集下游信号分 子 TRAFs 上与 CD40 非常相似, 但是 LMP1 也不能完 全取代 $\mathrm{CD} 40^{[17]}$. 由此提示, 两者在某些方面还有差 异. 而且业已证实，当细胞受到 CD40L 配基化后， LMP1 和 CD40 会汇聚到一起，形成脂筏形式的信号 传导复合体, 向细胞内传递信号 ${ }^{[18]}$. 仅仅只有 LMP1 不足以维持 $\mathrm{B}$ 细胞在体外增殖. 因此, CD40 和 CD40L 在转化过程中很可能都是必需的.

通过流式细胞仪、RT-PCR 和 Western blotting 的检测，还显示 RPMI8226 细胞本身存在少量的 CD40L 的组成性表达，这与某些 B 淋巴瘤上有 CD40 和 CD40L 共表达的文献报道是一致的 ${ }^{[19]}$.

已有文献报道 ${ }^{[20,21]}, \mathrm{EB}$ 病毒体外感染正常 B 淋 巴细胞可以上调表达 CD40L, 而正是这些 CD40L 分 子在 $\mathrm{B}$ 细胞转化成永生化淋巴细胞系(LCL)过程中起 了重要作用. RPMI8226 细胞中上调表达的 CD40L 也 可能与感染细胞抗调亡有关 ${ }^{[22]}$. 细胞周期分析中发 现, 感染后 $24 \mathrm{~h}$ 细胞出现了调亡峰, 可能由于细胞短 
时间内受到病毒大剂量冲击有关. 而在感染后 $48 \mathrm{~h}$, 也就是蛋白水平 $\mathrm{CD} 40 \mathrm{~L}$ 上调时, 调亡峰消失(图 4C). 类似的结果在 Annexin- $V$ 和 PI 双标记的调亡检测中 也进一步得到证实(图 5). 然而, 感染后细胞 CD40 表 达下调(图 2C). 有研究发现 ${ }^{[23]}$, 低剂量 CD40 抗体可 以诱导白细胞介素-10 生成, 而高剂量时诱导白细胞 介素-12 生成. 我们前期研究也发现 ${ }^{[24,25]}$, 低剂量 CD40 抗体可以激发多发性骨髓瘤细胞增殖, 而高剂 量时诱导骨髓瘤细胞凋亡.

通过恶性转化可以使一些细胞的正常的生理机 制发生改变, 从而有利于肿瘤细胞的存活、生长和增 殖 ${ }^{[26]}$. 本研究发现, B 淋巴瘤细胞株 Daudi 和 Raji 可 以通过 CD40L 异位表达, 使 CD40 和 CD40L 在细胞 表面共表达, 并且共聚集, 有可能组成一个信号转导 复合体, 而利用阻断性 CD40mAb 或 CD40LmAb, 可 能通过以下两个方式阻断 CD40-CD40L 信号转导: (1) 破坏单个细胞表面信号传导复合体结构; (2) 阻断了 肿瘤细胞与细胞间 CD40-CD40L 的信号作用, 使肿 瘤细胞生长、增殖受到抑制, 并使细胞调亡率增加. 而且由于不同的抗体阻断的分子不同, 可以产生不 同程度的阻断效应. 如果阻断信号是以第一种方式 占优势, 那么阻断信号传导复合体中的任何一分子,
都有可能破坏复合体的完整性结构, 起到阻断信号 传导的作用. 因此临床上选用 CD40 作为靶分子进行 肿瘤治疗时，可以尝试考虑以 CD40 信号传导复合体 作为靶目标.

肝移植术后并发 $\mathrm{MM}$ 细胞被 $\mathrm{EB}$ 病毒感染后导致 的 CD40L 上调表达可能参与了浆细胞转变成恶性骨 䯣瘤细胞的过程和感染后细胞抗调亡效应. 而且, 趋 化因子受体 CXCR4 也在感染后明显上调表达(图 2D), 细胞迁徙率也显著提高, 使得感染细胞迁徙能力增 加, 这也是肿瘤恶性程度的一个重要特征. CD40 和 $\mathrm{CD} 40 \mathrm{~L}$ 或 EBV 抗原 LMP1 可以在感染后的细胞表面 共聚集, 无论是骨髓瘤新鲜标本, 还是 B 淋巴瘤细胞 株 Daudi 或 Raji，都有 CD40 和 CD40L 共表达现象, 并且两者可以在细胞表面共聚集. CD40 和 CD40L 共 表达是有意义的，用阻断性 CD $40 \mathrm{mAb}$ 或 CD40LmAb 阻断 CD40-CD40L 信号, 能显著抑制 Daudi 或 Raji 细胞生长、增殖, 并诱导细胞凋亡; 不同抗体由于结 合分子不同，对信号阻断的强度是不同的，并且呈抗 体剂量依赖性.

有关 EB 病毒导致的疾病与 CD40-CD40L 的关系, 还有待进一步研究，其意义在于将为今后的肿瘤预 防和免疫治疗提供新途径.

\section{参考文献}

1 Berenson J R, Vescio R A. HHV-8 Is present in multiple myeloma patients. Blood, 1999, 93: 3157-3159

2 Bishop G A, Hostager B S. The CD40-CD154 interaction in B cell-T cell liaisons. Cytokine Growth Factor Rev, 2003, 14: 297-309

3 Busch L K, Bishop G A. The EBV transforming protein, LMP1, mimics and cooperates with CD40 signaling in B lymphocyte. J Immunol, 1999, 162: 2555-2561

4 Cacciarelli T V, Green M, Jaffe R, et al. Management of posttransplant lymphoproliferative disease in pediatric liver transplant recipients receiving primary tacrolimus (FK506) therapy. Transplantation, 1998, 66: 1047-1052

5 Challa A, Eulopulos A G, Holder M J, et al. Population depletion activates autonomous CD154-dependent survival in biopsy-like Burkitt's lymphoma cells. Blood, 2002, 99: 3411-3418

6 Clodi K, Asgary Z, Zhao S, et al. Coexpression of CD40 and CD40 ligand in B-cell lymphoma cells. Br J Haematol, 1998, 103: 270-275

7 Fuleihan R, Ramesh N, Horner A, et al. Cyclosporin A inhibits CD40 ligand expression in T lymphocytes. J Clin Invest, 1994, 93: 1315-1320

8 Hallek M, Bergsagel P L, Anderson K C. Multiple myeloma: Increasing evidence for a multistep transformation process. Blood, 1998, 91: $3-21$

9 Hanissian S H, Geha R S. Jak3 is associated with CD40 and is critical for CD40 induction of gene expression in B cells. Immunity, 1997, 6: 379-387

10 Imadome K, Shirakata M, Shimizu N, et al. CD40 ligand is a critical effector of Epstein-Barr virus in host cell survival and transformantion. Proc Natl Acad Sci USA, 2003, 100: 7836-7840

11 Kaykas A, Worringer K, Sugden B. CD40 and LMP-1 both signal from lipid rafts but LMP-1 assembles a distinct, more efficient signaling complex. EMBO J, 2001, 20: 2641-2654

12 Oh H M, Oh J M, Choi S C, et al. An efficient method for the rapid establishment of Epstein-Barr virus immortalization of human B 
lymphocytes. Cell Prolif, 2003, 36: 191-197

13 Larsen C P, Elwood E T, Alexander D Z, et al. Long-term acceptance of skin and cardiac allografts after blocking CD40 and CD28 pathways. Nature, 1996, 381: 434-438

14 Arcipowski K M, Stunz L L, Graham J P, et al. Molecular mechanisms of TNFR-associated factor 6 (TRAF6) utilization by the oncogenic viral mimic of CD40, latent membrane protein 1 (LMP1). J Biol Chem, 2011, 286: 9948-9955

15 Wang Z, Yang S, Zhou L, et al. Specific cellular immune responses in mice immunized with DNA, adeno-associated virus and adenoviral vaccines of Epstein-Barr virus-LMP2 alone or in combination. Sci China Life Sci, 2011, 54: 263-266

16 Mathur R K, Awasthi A, Wadhone P, et al. Reciprocal CD40 signals through p38MAPK and ERK-1/2 induce counteracting immune responses. Nat Med, 2004, 10: 540-544

17 Mutimer D, Kaur N, Tang H, et al. Quantification of Epstein-Barr virus DNA in the blood after adult liver transplantation. Transplantation, 2000, 69: 954-959

18 Nalesnik M A, Rao A S, Furukawa H, et al. Autologous lymphokine-activated killer cell therapy of Epstein-Barr virus-positive and -negative lymphoproliferative disorders arising in organ transplant recipients. Transplantation, 1997, 63: 1200-1205

$19 \mathrm{Xu} \mathrm{H}$, Zhao G, Huang X, et al. CD40-expressing plasmid induces anti-CD40 antibody and enhances immune responses to DNA vaccination. J Gene Med, 2010, 12: 97-106

20 Qi C J, Zheng L, Zhou X, et al. Cross-linking of CD40 using anti-CD40 antibody, 5C11, has different effects on XG2 multiple myeloma cells. Immunol Lett, 2004, 93: 151-158

21 The International Myeloma Working Group. Criteria for the classification of monoclonal gammopathies, multiple myeloma and related disorders: a report of the International Myeloma Working Group. Br J Hematology, 2003, 121: 749-757

22 Tong A W, Seamour B, Chen J, et al. CD40 Ligand-induced apoptosis is fas-independent in human multiple myeloma cells. Leuk Lymphoma, 2000, 36: 543-558

23 Uchida J, Yasui T, Takaoka-Shichijo Y, et al. Mimicry of C40 signals by EBV LMP1 in B lymphocyte responses. Science, 1999, 286: 300-303

24 Young L S, Rickinson A B. Epstein-Barr virus: 40 years on. Nat Rev Cancer, 2004, 4: 757-768

25 Zimber-Strobl U, Kempkes B, Marschall G, et al. Epstein-Barr virus latent membrane protein (LMP1) is not sufficient to maintain proliferation of B cells but both it and activated CD40 can prolong their survival. EMBO J, 1996, 15: 7070-7078

26 Cao J, Shen C, Zhang J, et al. Comparison of alternative extraction methods for secretome profiling in human hepatocellular carcinoma cells. Sci China Life Sci, 2011, 54: 34-38 